\title{
ERRATUM
}

Mathilde Dufaÿ • Martine Hossaert-McKey •

Marie-Charlotte Anstett

\section{Temporal and sexual variation of leaf-produced pollinator-attracting odours in the dwarf palm}

Published online: 9 June 2004

(C) Springer-Verlag 2004

\section{Oecologia (2004) 139:392-398}

In the pdf and print versions, the author's name Dufaÿ was incorrectly spelled Dufa. The following references and text citations were also affected:

\section{References}

Dufaÿ M, Anstett M-C, Hossaert-McKey M (2003) When leaves act like flowers: how dwarf palms attract their pollinators. Ecol Lett 6:28-34

Dufaÿ M, Anstett M-C (2004) Cheating is not always punished: killer female plants and pollination by deceit in the dwarf palm Chamaerops humilis. J Evol Biol (in press)

The online version of the original article can be found at http://dx. doi.org/10.1007/s00442-004-1538-y

M. Dufaÿ $(\bowtie) \cdot$ M. Hossaert-McKey $\cdot$ M.-C. Anstett

CNRS-CEFE (Centre d'Ecologie Fonctionnelle et Evolutive),

1919 Route de Mende,

34293 Cedex 05 Montpellier, France

e-mail: mathilde.dufay@univ-lille1.fr

Tel.: +33-3-20335923

Fax: +33-3-20436979

Present address:

M. Dufaÿ

Génétique et Evolution des Populations Végétales, Université

des Sciences et Techniques de Lille,

Batiment SN2,

59655 Cedex Villeneuve d'Ascq, France 